\title{
Effect of size on mechanical behavior of Au pillars by molecular dynamics study
}

\author{
TANG QiHeng ${ }^{1,2 *}$ \\ ${ }^{1}$ State Key Laboratory of Nonlinear Mechanics, Institute of Mechanics, Chinese Academy of Sciences, Beijing 100190, China; \\ ${ }^{2}$ State Key Laboratory of Explosion Science and Technology, Beijing Institute of Technology, Beijing 100081, China
}

Received February 7, 2012; accepted March 16, 2012; published online April 17, 2012

\begin{abstract}
The influence of specimen size on the mechanical behavior of Au pillars is studied by means of molecular dynamics (MD) simulations with the EAM potential. Under compression at $300 \mathrm{~K}$, as the deformation of pillars is in the plastic stage, nucleation of partial dislocations is observed. The coupling effect of surface stress and thermal activation is considered when analyzing the size effect on the yield property of the Au pillars. It appears that both the tensile stress component and the temperature in the surface layer impart significant effect on the mechanical behaviors of the nano-sized Au pillars.
\end{abstract}

molecular dynamics simulation, size effect, dislocation, compression

PACS number(s): 61.46.Hk, 61.72.Bb, 47.11.Mn

Citation: Tang Q H. Effect of size on mechanical behavior of Au pillars by molecular dynamics study. Sci China-Phys Mech Astron, 2012, 55: 1111-1117, doi: $10.1007 / \mathrm{s} 11433-012-4740-7$

In recent years there has been an increased focus in the specimen size effects on solids. Such effects have been established in various metals and alloys under tension, torsion, indentation as well as compression [1-3]. Various theories have been proposed and articulated to explain size effect under torsion and indentation in regard to strain gradient plasticity [4-7]. Recently focus has been given to the size effect of single crystal metals under compression because of the advent of focused ion beam (FIB) technique that allows the fabrication of pillars to small sizes (pillars with diameter $<100 \mathrm{~nm}$ that can be manufactured with FIB). It has been argued that under micro-tension, strain gradient effect is absent, and thus an alternative theory has to be suggested to explain the strong effect of pillar size on the plastic behavior of single crystal metals. In situ TEM by Shan et al. [8] has substantiated the idea that source starvation might be the mechanism responsible for the strong size effect under micro- and nano-compression of single crystal metals. The

*Corresponding author (email: qhtang@imech.ac.cn) strong interest in size effect is justified by the importance of the knowledge of the mechanical behaviors of small-size features because it is imperative to the development of nano-electro-mechanical systems (NEMS) and micro-electro-mechanical systems (MEMS) $[9,10]$.

Greer and Nix [11] investigated the plastic deformation of free-standing single crystal $\mathrm{Au}$ pillars by both experimental and molecular dynamics (MD) simulations. Their results indicate that the flow stress of these pillars exhibits strong size effects and increases significantly with decreasing pillar diameter at the sub-micro scale. They proposed an explanation for their observations based on dislocation starvation in that dislocations can easily exit the pillar surface before multiplication mechanisms such as cross-slip and dislocation-dislocation interactions commence. As a consequence, high stresses are required in order to nucleate new dislocations and to continue the plastic deformation processes [12-15]. The dislocation starvation mechanism has been supported by discrete dislocation analysis [16-18]. Frick et al. [19] performed experiments to investigate the 
uniaxial compression behavior of FIB manufactured [111] Ni pillars, with pillar diameters ranging from approximately $25 \mu \mathrm{m}$ to below $200 \mathrm{~nm}$. Their results also revealed a strong increase in yield stress and work hardening with decreasing pillar diameter.

Atomistic simulations have been employed to investigate the plastic deformation mechanisms with respect to size effect in nanometer scale materials [20-25]. Horstemeyer et al. [20] studied the length and time scale effects on the plastic flow of face-centered cubic (FCC) metals under simple shear loading. Others [21] investigated the mechanical responses of Au nanowires subjected to uniaxial compression and discussed the effects of size and slenderness ratio on the mechanical properties. Some researchers have studied the size and strain rate effects in tensile deformation of copper nanowires [22]. Yang et al. [23] and Liang et al. [24] revealed an increase in the tensile yield stress of metal nanowires with a decreasing cross-sectional area, and von Mises yield condition was used to analyze the simulation results in literature [23]. Diao et al. [25] employed MD to study the effect of specimen size on the yielding behavior of $\mathrm{Au}$ nanowires with a width less than $6 \mathrm{~nm}$. The results showed that the compressive yield stress becomes more pronounced with increasing nanowire width, which is in contrast to the other results [11]. The contradiction may be attributed to different plastic deformation mechanism. By using the concept of critical resolved shear stress, Diao et al. [25] proposed a local yield criterion to explain the observed size dependence of the yielding behavior. Since nanowires with diameter less than $6 \mathrm{~nm}$ have a large surface-area/ volume ratio as compared to sub-micro specimens, it should not be unduly expected to find a strong surface effect. They also noted out those free surfaces are critical in the yielding of nanowires. Firstly they act as preferential nucleation sites for defects and secondly the surface stresses can exist on the surface of a wire, and such stresses may induce the yielding of the nanowire. These characteristics may also be observed on the nano-pillars. The details of the dislocation nucleation from the surface by MD simulations are usually carried out at pronounced strain rates [26,27]. However, Zhu et al. [28] developed an atomistic modeling framework to address the surface dislocation nucleation. That is, a small activation volume led to the increased strain-rate and temperature sensitivities of flow stress. Hence, the nucleation stress over a large range of temperatures and strain rates may be able to be predicted [29].

Though many researchers have uncovered interesting observations in regard to the deformation mechanisms such as the yield behavior on the nanometer-scale specimens of single crystal metals, there is yet no comprehensive understanding available of the mechanism leading to the observed behavior. The objective of this work is to use atomistic simulation to quantitatively study the size effect of nanometer pillars of the single crystal $\mathrm{Au}$ under uniaxial compressive loading. Specifically we have taken into account the cou- pling effect of surface stresses and temperature in the surface layer in order to provide a new interpretation regarding the size dependence of plastic deformation on the nanometer scale.

\section{Computation and modeling}

\subsection{Interatomic potential}

It is known that the accuracy of MD simulations is dictated by the accuracy of the interatomic potentials being used. The embedded atom method (EAM) to describe potential for FCC Au [30] is adopted. In this context, the total energy of the system is given by

$$
E_{T}=\sum_{i=1}^{N} F_{i}\left(\rho_{i}\right)+\frac{1}{2} \sum_{i j, i \neq j} \phi_{i j}\left(r_{i j}\right)
$$

where $\phi_{i j}\left(r_{i j}\right)$ is the pair interaction and $r_{i j}$ is the distance between atoms $i$ and $j, F_{i}$ is the embedding function and $\rho_{i}$ is the electronic density at the site of atom $i$. Most simulation results show that the EAM potential is valid for metallic materials. All parameters for the interatomic potential of Au can be found from [30]. The components of the stress tensor associated with an atoms is defined as $[31,32]$ :

$$
\sigma_{k l}=\frac{1}{\Omega_{0}}\left(-\frac{p_{k} p_{l}}{m}+\sum_{j \neq i} \frac{1}{2} \frac{\partial \phi}{\partial r} \frac{r_{i j}^{k} r_{i j}^{l}}{r_{i j}}\right),
$$

where $\sigma_{k l}$ is the stress component, $\mathrm{p}$ is the atomic momentum, $\mathrm{m}$ is the atomic mass, $r_{i j}^{k}$ and $r_{i j}^{l}$ are the k-th, 1-th components of $r_{i j}$, and $\Omega_{0}$ is the volume of an atom.

\subsection{Models of nano-pillars}

Pillar samples to be used in the MD simulations are ideal and dislocation-free Au single crystal. Diameters of the pillars range from $5 \mathrm{~nm}$ to $15 \mathrm{~nm}$. Figures 1(a) and 1(b) are the three-dimensional atomic system model and the profile of a cross section along the pillar axis. The pillar diameter $d$ is measured at the half height of the pillar. The aspect ratio (height to diameter) is 4 , and the taper angle is about $2.86^{\circ}$. The geometry of the pillars modeled in this work is comparable to those experimentally investigated by Greer and Nix [11], but the pillar size of the present work is smaller.

The MD simulations are performed under the NVT ensemble. Initial velocities of atoms are specified based on the Maxwellian distribution corresponding to $T=300 \mathrm{~K}$, and the magnitudes may be adjusted to permit the temperature constant in the system according to the Nose-Hoover thermostat $[33,34]$. The time integration of motion is performed by using the velocity-Verlet algorithm [35].

To simulate the compressive load acting on the two ends 
of the pillar along its axis direction, the $x$ and $y$ displacements of top two layers and bottom two layers displacements were constrained, while their movement in the $z$-axis ([001]) at an average loading rate of $5.0 \times 10^{8} \mathrm{~s}^{-1}$ was prescribed. The pillar side surface was free. After each loading step, 1000 steps of MD relaxation at the constant temperature of $300 \mathrm{~K}$ were performed, so that the equilibrium state of atoms could be reached.

According to the geometric structure of a nano-pillar, it can be divided into two regions: the first is the surface layer, and second is the interior region. Suppose $N_{\mathrm{t}}$ is the total number of atoms, that is, $N_{\mathrm{t}}=N_{\mathrm{s}}+N_{\mathrm{i}}$, where $N_{\mathrm{s}}$ and $N_{\mathrm{i}}$ are the numbers of the surface atoms and the interior atoms, respectively. The surface atom fraction is the ratio of $N_{\mathrm{s}} / N_{\mathrm{t}}$, and the interior atom fraction is the ratio of $N_{\mathrm{i}} / N_{\mathrm{t}}$.

For simplification in the following analysis, we first define the following quantities:

$$
\begin{gathered}
\sigma_{k l}=\sum_{n=1}^{N \mathrm{t}} \sigma_{k l, n} / N_{\mathrm{t}}, \\
\sigma_{k l, s p}=\sum_{n=1}^{N \mathrm{~s}} \sigma_{k l, n} / N_{\mathrm{t}}, \\
\sigma_{k l, i p}=\sum_{n=1}^{N \mathrm{i}} \sigma_{k l, n} / N_{\mathrm{t}},
\end{gathered}
$$

where $\sigma_{k l}$ is the average stress, $\sigma_{k l, s p}$ and $\sigma_{k l, i p}$ are termed the average surface stress portion and average interior stress portion, respectively, and $\sigma_{k l, n}$ is the stress of atom $\mathrm{n}$, and $\mathrm{k}$ and $\mathrm{l}$ are the coordinate directions. Based on above definitions, one can derive $\sigma_{k l}=\sigma_{k l, s p}+\sigma_{k l, i p}$, which correlates to the average stress per atom consisting of two parts: the surface stress portion and the interior stress portion.

(a)

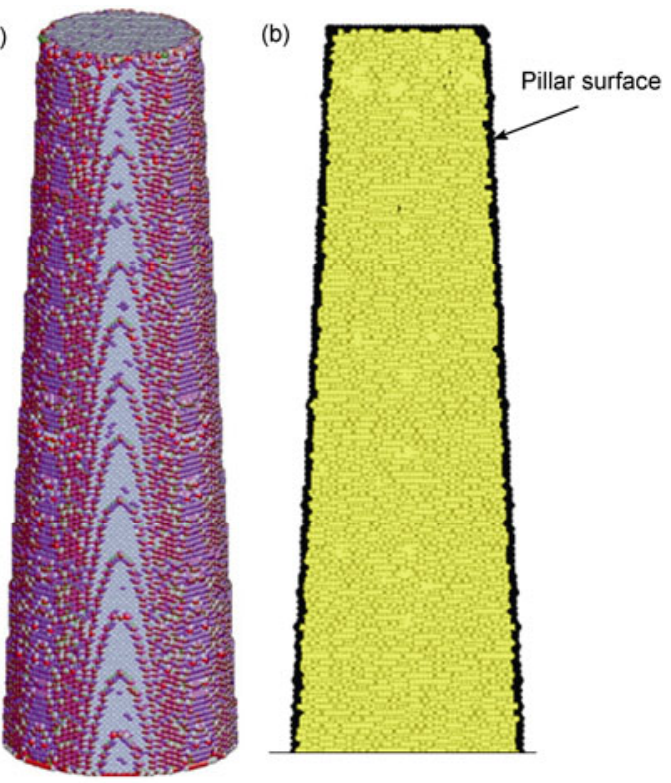

Figure 1 (Color online) Atom configuration after relaxation. (a) Threedimensional; (b) diagram of the cross section.

\section{Results and discussion}

\subsection{Plastic deformation mechanism at atomic scale}

Before relaxation, pillars carved from the block have perfect lattice in the interior, but the surface atoms are in close proximity to atoms without symmetry, and as a consequence they are in a tensile stress state.

Relaxation is carried out with temperature of $300 \mathrm{~K}$, and a stable atomic system is obtained after $10000 \mathrm{MD}$ steps at a time interval $\Delta t=2 f s$. By comparing with the ideal crystal structure, the surface atoms lose some of their neighboring atoms, thus there will be an unbalanced force acting on these atoms, which leads to the tensile stress on the surface and compressive stress in the interior. The whole atomic system will reach equilibrium gradually with the process of relaxation.

A small amount of compressed deformation is observed by examining the motion of the surface atoms. It is observed that the pillar surface is no longer smooth upon deformation, and some defects appear on the surface. The coordination number of the surface atoms is less than 12 for a perfect FCC lattice.

Figure 2(a) shows the potential energy plotted versus MD steps of nanopillars after NVT relaxation. It can be seen that the system can reach the stable state after 1000 MD steps. The average potential energy of per atom is about $-3.854 \mathrm{eV}$. The potential energy on the surface is a slightly more evident than that within the interior. Figure 2(b) shows the kinetic energy plotted versus MD steps. It seems that the kinetic energy on the surface is more significant than that of the interior region.

After relaxation, the average stresses per atom in the interior and surface are about $-2.23 \mathrm{GPa}$, and $2.3 \mathrm{GPa}$, respectively. Similar results are obtained in the MD simulations of Au nanowires by Diao et al. [25].

Koh and Lee [36] proposed that the presence of tensile stresses in the surface of nanometer materials is attributed to the availability of surface atoms at a higher electronic energy state as compared to atoms situated within the interior of the material. This may result in surface tension because of formation of asymmetric bonding of surface atoms with neighboring atoms.

The slip systems of $\langle-112\rangle /(1-11)$ of FCC metal are selected to study the yielding behavior of the nano-pillars of Au. Figure 3(a) shows the initial relaxed atomic configuration at zero compressive strain, which shows a consistent periodic atomic structure with stacking sequence of ABCABC... along [1-11] direction. As the compressive strain reaches $4 \%$, the deformation is still elastic with no dislocations nucleated, as shown in Figure 3(b). As the compressive strain reaches $5 \%$, partial dislocations begin to appear, as observed in Figure 3(c), on the slip plane of (1-11), and the partial dislocation nucleated and extended 

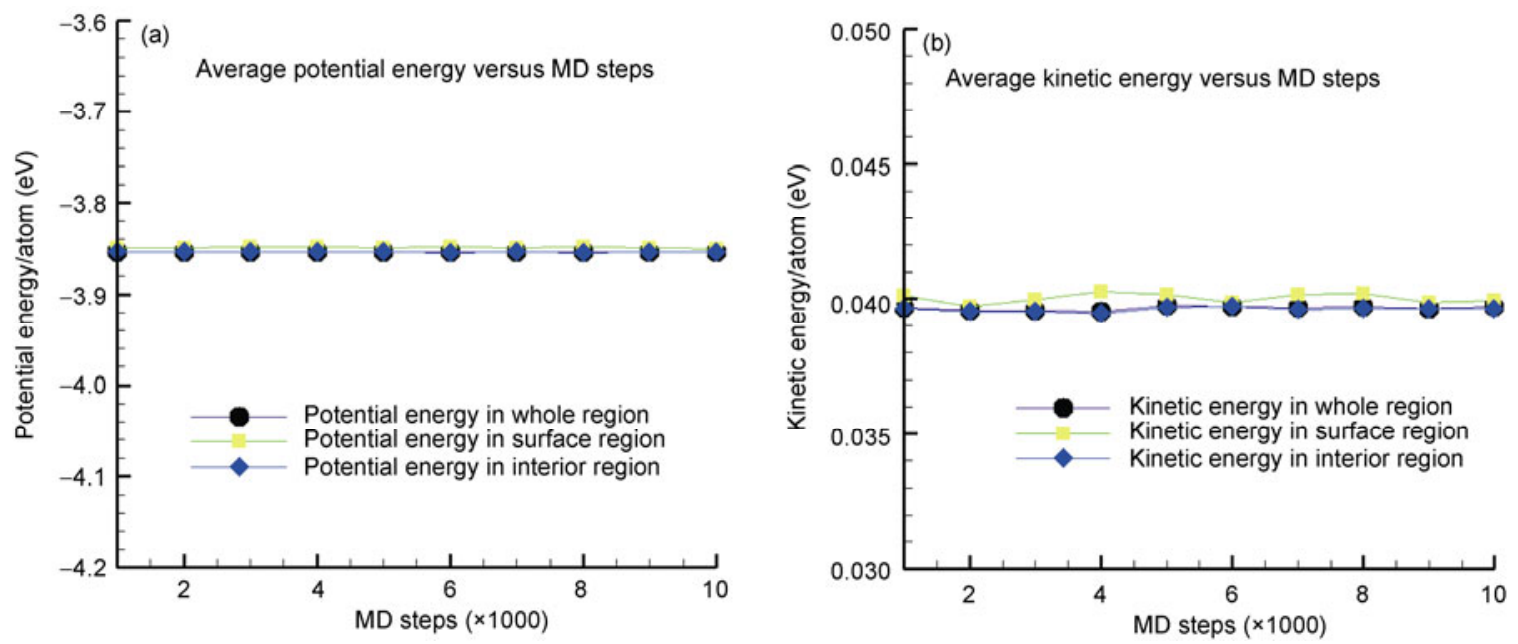

Figure 2 Average energy per atom versus MD steps during NVT relaxation. (a) Average potential energy per atom versus MD steps; (b) average kinetic energy per atom versus MD steps.
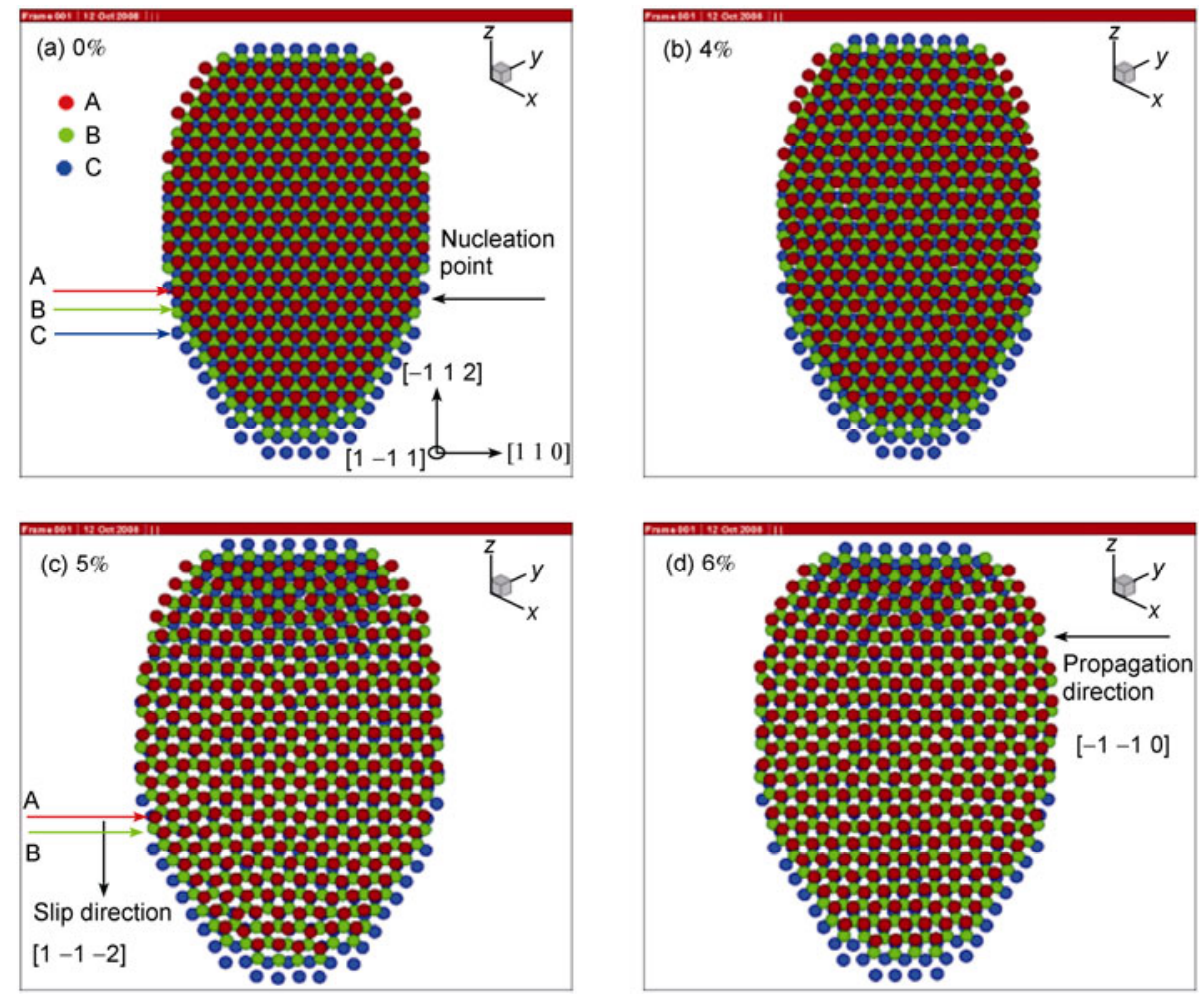

Figure 3 Atom configuration on slip plane (1-11). (a) 0 strain; (b) strain=4\%; (c) strain=5\%; (d) strain=6\%.

in the [-112] direction. At this point, the initially periodic structure of the atomic system becomes one with a stacking fault defect. Finally, when the compressive strain is $6 \%$ more stacking faults are observed as is shown in Figure $3(d)$.

Let us take a pillar with diameter $12.5 \mathrm{~nm}$ for a more detailed analysis. The stress-strain curve of this nano-pillar is plotted in Figure 4. As the total strain reaches 4\%, the stress $\sigma_{z z}$ is about $-0.55 \mathrm{GPa}$. No defects are observed, implying that the compressed deformation is still within the elastic range (Figure 3(a)). This is further verified by the atomic configuration shown in Figure 3(b). As the total strain reaches $5 \%$, the stress $\sigma_{z z}$ increases to $-0.72 \mathrm{GPa}$. The corresponding atomic arrangements are shown in Figure 3(b). The resolved shear stress acting on the slip system $\langle-112\rangle /(1-11)$ is $\tau=\sigma_{z z} \cdot m . m$ is Schmidt factor and $m=$ $\cos \alpha \cos \beta$, where $\alpha$ is angle between two vectors $\langle 1-11\rangle$ and $\langle 001\rangle$, and $\beta$ is angle between $\langle-112\rangle$ and $\langle 001\rangle$, here 


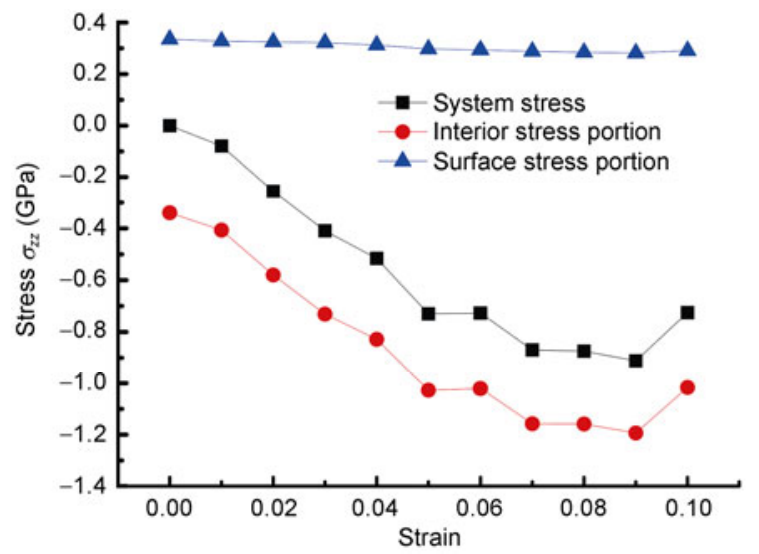

Figure 4 (Color online) Curve of the stress portion versus strain for diameter $12.5 \mathrm{~nm}$.

$m$ is about 0.471 . It is the shear stress $\tau$ which led to dislocation nucleation. We see here that plastic yielding has occurred as a several slip systems have been activated, in accordance with the analysis of Figure 3(c). As strain is 6\%, the stress $\sigma_{z z}$ is about $-0.69 \mathrm{GPa}$, and the stress-strain curve begins to level. This is attributed to activation of more slip systems, which also leads to the creation of more defects. The atom configuration (c) in Figure 3 shows clearly the generation of more defects. After the compressive strain reaches $9 \%$, the stress has dropped greatly, such that the pillar has lost its ability to bear the applied load.

As the stress-strain curve (Figure 4) indicates, evolution of interior stress portion follows the same pace as that of the average (applied) stress. The interior stress portion increases with strain in the elastic deformation regime. After the yielding of the pillar, the interior stress portion exhibits some variation. This is an interesting observation which has been established also for pillar sizes, from $5 \mathrm{~nm}$ to $15 \mathrm{~nm}$. It could be interpreted that as nano-pillar of $\mathrm{Au}$ is going through plastic flow, the surface stress portion shows no significant variation. This is unlike the interior stress portion which can be used to characterize the various stages of plastic deformation.

\subsection{Size effect on the mechanical behavior of nano- pillars of $\mathrm{Au}$}

To study the size effect on the mechanical behavior at nanometer scale, MD simulations were used to investigate the behaviors of five nano-pillars of under compressive load. The diameters of the nano-pillars are $5 \mathrm{~nm}, 7.5 \mathrm{~nm}, 10 \mathrm{~nm}$, $12.5 \mathrm{~nm}$ and $15 \mathrm{~nm}$, respectively.

Figure 5 shows the yield stresses of the pillars as a function of the pillar diameter. The yield stress increases with pillar size. Also shown in Figure 5 are the surface stress portion and the interior stress portion versus pillar sizes. Upon yielding, both the interior stress portion and the surface stress portion are sensitive to the pillar size. As the

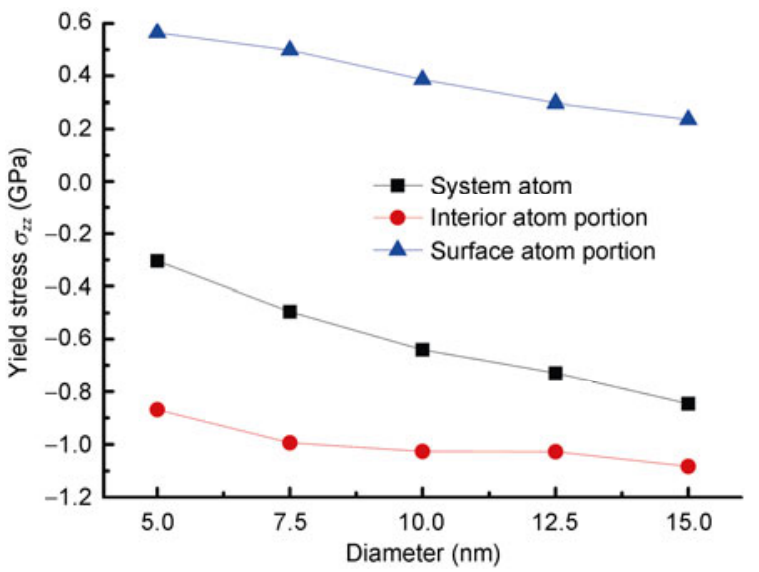

Figure 5 (Color online) Curve of the yield stress versus sizes.

pillar diameter increases, the influence of the surface stress portion decreases. If the pillar diameter is sufficiently large, the surface stress portion approaches zero, implying that the influence of pillar surface on the mechanical behavior can be neglected.

Combining Figures 3(c) and 3(d), one can observe that the slip direction of the leading partial dislocation is along [1-1-2] on the slip plane (1-11), which is consistent with direction of interior shear stress. Based on our simulation a suggestion is proposed that the interior shear stress state is vital to the yielding behavior of pillar Au. Diao et al. [25] performed MD simulation of nanowire and a similar result was obtained. From Figure 5 it can be observed that as the pillar diameter decreases, the surface stress portion increases markedly. If the pillar diameter is $7.5 \mathrm{~nm}$, the surface stress reaches $-0.525 \mathrm{GPa}$, the negative value meaning that the surface layer is in the tension state. The tensile stress state in the surface layer is quite remarkable at the nanometer scale. The evolution of the surface stress portion with the pillar sizes is similar to that of the yield stress, indicating that the yielding is sensitive to the stress state of the surface layer.

MD simulations of the five pillars with different pillar sizes show that the interior atoms are in a compressive state, whereas the surface atoms are in a tensile state. This suggests that the lattice parameter of the interior is $a_{0}-\varepsilon$, where $a_{0}$ and $\varepsilon$ are the equilibrium (stress-free) lattice constant and a small quantity, respectively. On the contrary, the lattice parameter of the surface layers should be $a_{0}+\varepsilon$. That is to say, the interior atoms are subjected to constraint by the surrounding atoms, and their imposed velocity may thus be relatively small. Surface atoms are subject to less constraint, resulting in a higher imposed velocity. This notion has been substantiated by MD simulation. Based on statistic thermal analysis, the temperature of an atomic system is dependent on atomic velocity. Rice and Beltz [37] and Meyers and Chawla [38] pointed out that the effects of thermal activation are significantly lower for the load of dislocation nu- 
cleation. The kinetics of dislocation nucleation can be described by the Arrhenius type equation [38]. Combining the concept of thermal activation energy with MD simulations, Kitagawa et al. [39] as well as Zhang et al. [40] revealed that the critical stress intensity factor $K_{c r}$ for dislocation nucleation at a crack tip decreases with temperature, and thermal fluctuations within a system may assist atoms in overcoming the potential barrier and generate dislocations. Recently, researchers have developed an atomistic modeling framework to address the probabilistic nature of surface dislocation nucleation [28]. The results show that the nucleation of surface dislocation is sensitive to temperature and the applied load required for dislocation nucleation is reduced with temperature.

Experimentally, dislocations are frequently observed to nucleate at material surface. We believe, based on our MD simulations, the thermal activation may be a critical mechanism because the surface temperature is higher than that of the interior region. Therefore, the thermal activation should also assist us to understand the size effect on yielding behavior of nanometer materials.

\section{Conclusions}

MD simulations of five nano-pillars of $\mathrm{Au}$ with different pillar sizes are carried out in order to understand the size effect on the yielding behavior of nanometer materials. By combining the analysis of thermal activation with both evolutions of the surface stress, our work reveals the mechanism of plastic deformation at nanoscale.

For a typical pillar with diameter of $12.5 \mathrm{~nm}$, as strain is $5 \%$, the nucleation of partial dislocation is observed, and the yield stress reaches $-0.72 \mathrm{GPa}$.

The thermal activation is important in lowering the external load required for dislocation nucleation. MD simulations show that the surface temperature is higher than that of interior region, and this result may explain the frequent observations that dislocations are nucleated at material surfaces.

The MD simulations show that as the pillar size decreases the yield stress also decreases, which is accompanied by great increase in the surface stress of the nanopillars.

This work was supported by the National Natural Science Foundation of China (Grant Nos. 10872197, 11021262, 11172303, 11132011).

1 Nix W D, Gao H J. Indentation size effects in crystalline materials: A law for strain gradient plasticity. J Mech Phys Sol, 1998, 46: 411-425

2 Ma Q, Clarke D R. Size-dependent hardness of silver single-crystals. J Mater Res, 1995, 10: 853-863

3 Uchic M D, Dimiduk DM, Florando J N, et al. Sample dimensions influence strength and crystal plasticity. Science, 2004, 305: 986-989
4 Fleck N A, Muller G M, Ashby M F, et al. Strain gradient plasticity-theory and experiment. Acta Metall Mater, 1994, 42: 475-487

5 Fleck N A, Hutchinson J W. Strain gradient plasticity. Adv Appl Mech, 1997, 33: 295-361

6 Gao H, Huang Y, Nix W D, et al. Mechanism-based strain gradient plasticity - I. Theor J Mech Phys Sol, 1999, 47: 1239-1263

7 Hwang K C, Jiang H, Huang Y, et al. A finite deformation theory of strain gradient plasticity. J Mech Phys Sol, 2002, 50: 81-99

8 Shan Z W, Mishra R K, Asif S A, et al. Mechanical annealing and source-limited deformation in submicrometre-diameter Ni crystals. Nat Mater, 2008, 7: 115-119

9 Craighead H G. Nanoelectromechanical system. Science, 2000, 290: 1532-1535

10 Komanduri R, Chandrasekaran N, Raff L M. Molecular dynamics simulation of uniaxial tension of some single-crystal cubic metals at nanolevel. Int J Mech Sci, 2001, 43: 2237-2260

11 Greer J R, Nix W D. Size dependence of mechanical properties of gold at the sub-micron scale. Appl Phys A, 2005, 80: 1625-1629

12 Volkert C A, Lilleodden E T. Size effects in the deformation of sub-micron Au columns. Philos Mag A, 2006, 86: 5567-5579

13 Greer J R, Nix W D. Nanoscale gold pillars strengthened through dislocation starvation. Phys Rev B, 2006, 73: 245410

14 Cao A, Wei Y, Mao S X. Alternating starvation of dislocations during plastic yielding in metallic nanowires. Scripta Mater, 2008, 59: 219-222

15 Greer J R, Oliver W C, Nix W D. Size dependence of mechanical properties of gold at the micron scale in the absence of strain gradients. Acta Mater, 2005, 53: 1821-1830

16 Balint D S, Deshpande V S, Needleman A, et al. Size effects in uniaxial deformation of single and polycrystals: A discrete dislocation plasticity analysis. Model Simul Mater Sci Eng, 2006, 14: 409-422

17 Benzerga A A, Shaver N F. Scale dependence of mechanical properties of single crystals under uniaxial loading. Scripta Mater, 2006, 54: 1937-1941

18 Tang H, Schwarz K W, Espinosa H D. Dislocation escape-related size effects in single-crystal micropillar under uniaxial deformation. Acta Mater, 2005, 55: 1607-1616

19 Frick C P, Clark B G, Orso S, et al. Size effect on strength and strain harding of small-scale [111] nickel compression pillars. Mater Sci Eng A, 2008,498: 319-329

20 Horstemeyer M F, Baskes M I, Plimpton S J. Length scale and time scale effects on the plastic flow of fcc metals. Acta Mater, 2001, 49: 4363-4374

21 Wen Y H, Wang Q, Liew K M, et al. Compressive mechanical behavior of Au nanowires. Phys Lett A, 2010, 374: 2949-2952

22 Liang W W, Zhou M. Size and strain rate effects in tensile deformation of Cu nanowires. Nanotechnology, 2003, 14: 452-455

23 Yang Z Y, Lu Z X, Zhao Y P. Atomistic simulation on size-dependent yield strength and defects evolution of metal nanowires. Comput Mater Sci, 2009, 46: 142-150

24 Kang J W, Hwang Y. Mechanical deformation study of copper nanowire using atomistic simulation. Nanotechnology, 2001, 12: 295-300

25 Diao J, Gall K, Dunn M L, Zimmerman J A. Atomistic simulations of the yielding of gold nanowires. Acta Mater, 2006, 54: 643-653

26 Diao J, Gall K, Dunn M L. Yield strength asymmetry in metal nanowires. Nano Lett, 2004, 4: 1863-1867

27 Rabkin E, Nam H S, Srolovitz D J. Atomistic simulation of the deformation of gold nanopillars. Acta Mater, 2007, 55: 2085-2099

28 Zhu T, Li J, Samanta A, et al. Temperature and strain-rate dependence of surface dislocation nucleation. Phys Rev Lett, 2008, 100: 025502

29 Mason J K, Lund A C, Schuh C A. Determining the activation energy 
and volume for the onset of plasticity during nanoindentation. Phys Rev B, 2006, 73: 054102

30 Foiles S M, Daw M S, Baskes M I. Embedded-atom-method functions for the fcc metals $\mathrm{Cu}, \mathrm{Ag}, \mathrm{Au}, \mathrm{Ni}, \mathrm{Pd}, \mathrm{Pt}$, and their alloys. Phys Rev B, 1986, 33: 7983-7991

31 Egami T, Maeda K, Vitek V. Structural defects in amorphous solids, a computer simulation study. Philos Mag A, 1980, 41: 883-901

32 Cheung K S, Yip S. Atomic-level stress in an inhomogeneous system. J Appl Phys, 1991, 70: 5688-5690

33 Nose S. A molecular dynamics method for simulations in the canonical ensemble. Mol Phys, 1983, 50: 1055-1076

34 Hoover W G. Canonical dynamics: equilibrium phase-space distributions. Phys Rev A, 1985, 31: 1695-1697

35 Leach A R. Molecular Modelling: Principles and Applications. Eng- land: Addison Wesley Longman, 1996

36 Koh S J A, Lee H P. Molecular dynamics simulation of size and strain rate dependent mechanical response of FCC metallic nanowires. Nanotechnology, 2006, 17: 3451-3467

37 Rice J R, Beltz G E. The activation energy for dislocation nucleation at a crack. J Mech Phys Sol, 1994, 42: 333-360

38 Merers M A, Chawla K K. Mechanical Metallurgy. Prentice Hall Inc, 1983

39 Kitagawa H, Nakatani A, Shibutani Y. Molecular dynamics study of crack processes associated with dislocation nucleated at the tip. Mater Sci Eng A, 1994, 176: 263-269

40 Zhang Y W, Wang T C, Tang Q H. The effect of thermal activation on dislocation processes at an atomistic crack tip. J Phys D-Appl Phys, 1995, 28: 748-754 